Artículo original

\title{
PREOCUPACIÓN AMBIENTAL Y SU RELACIÓN CON LA CONDUCTA ECOLÓGICA EN ESTUDIANTES DE INGENIERÍA DE LA UNIVERSIDAD PRIVADA DE TACNA
}

\author{
ENVIRONMENTAL CONCERN AND ITS RELATION TO ECOLOGICAL \\ BEHAVIOR IN ENGINEERING STUDENTS FROM THE PRIVATE \\ UNIVERSITY OF TACNA
}

Sebastián Martín Bernedo Berríos ${ }^{1}$

José Oswaldo Cazorla Galdos²

Información del artículo: Recibido: $13 / 07 / 2020$ Aceptado: 18/10/2020

${ }^{1}$ Escuela de Ingeniería Ambiental, Universidad Privada de Tacna, Perú ${ }^{2}$ Docente Escuela de Ingeniería Ambiental, Universidad Privada de Tacna, Perú E-mail: ${ }^{1}$ sebastian_1285@hotmail.com, 2jcazorlag@yahoo.com 


\section{Resumen}

El objetivo de la investigación fue determinar la relación entre la preocupación ambiental y la conducta ecológica en los estudiantes de la Facultad de Ingeniería de la Universidad Privada de Tacna, mediante un estudio descriptivo de nivel correlacional, se empleó la técnica de la encuesta utilizando como instrumento el cuestionario. la muestra de 310 estudiantes seleccionados al azar y de forma proporcional provino de las seis escuelas profesionales que componen la facultad, entre los resultados más destacados; se encontró que el 53,9\% de los estudiantes consideran que su nivel de preocupación por la problemática ambiental es regular, la dimensión más destacada fue el "Locus de control", donde los estudiantes consideran que su nivel de asumir el rol respecto a la crisis energética y ambiental es elevada, así mismo al comparar por escuelas profesionales; los estudiantes de Ingeniería Ambiental presentaron mayores valores medios; el 53,2 \% de los estudiantes afirmaron que su nivel de conducta ecológica, el cual que busca priorizar el cuidado del medio ambiente es regular, la dimensión más apreciable fue la "Conservación de la energía", destacando nuevamente los estudiantes de la escuela de Ingeniería Ambiental quienes presentaron los mayores valores en términos de media. Se concluye que existe relación directa y significativa (Rho de Spearman $=0,614$ y $p=0,000$ ) entre la preocupación ambiental y la conducta ecológica en estudiantes.

Palabras clave: Preocupación ambiental; conducta ecológica; problemas ambientales.

\section{Abstract}

The objective of the research was to determine the relationship between environmental concern and ecological behavior in students of the Faculty of Engineering of the Private University of Tacna, through a descriptive study of correlate level, the survey technique was used using the questionnaire as an instrument. the sample of 310 randomly and proportionally selected students came from the six professional schools that make up the faculty, among the most outstanding results; it was found that $53.9 \%$ of students consider their level of concern about environmental issues to be regular, the most prominent dimension being the "Control Locus", where students consider their level of taking on the role of the energy and environmental crisis to be high, as well as when comparing by professional schools; Environmental Engineering students had higher average values; $53.2 \%$ of students said that their level of ecological behavior, which seeks to prioritize environmental care is regular, the most appreciable dimension was "Energy Conservation", again highlighting the students of the School of Environmental Engineering who presented the highest values in terms of average. It is concluded that there is a direct and significant relationship (Spearman's Rho s 0.614 and $p \times 0.000$ ) between environmental concern and ecological behavior in students.

Keywords: Environmental concern; ecological behaviour; environmental problems. 


\section{Introducción}

A medida que transcurren los años, los seres humanos están tomando conciencia de los problemas ambientales que están generando en el planeta; al respecto, Scott y Willits (1994) y Dietz, Stern y Guagnano (1998) describen la creciente preocupación mundial por los daños en los ecosistemas, la contaminación del agua, el tratamiento inadecuado de los residuos sólidos, otros, que caracterizan los estilos de vida de las personas, es decir la existencia de los problemas ambientales tienen una directa relación con la forma de comportarse de las personas, y que a pesar de las diversas campañas para que las personas desarrollen una conciencia ambiental, no se ha logrado disminuir de forma significativa el impacto en el medio ambiente que genera las diversas actividades económicas, sociales, otros que desarrolla el ser humano.

En el Perú, existen serios problemas medio ambientales, de forma específica se tiene lo descrito por "Juntos por el Medio Ambiente" (Actualidad Ambiental. 2018, 6 febrero). Como una iniciativa que busca sensibilizar a los pobladores sobre los graves problemas ambientales existentes en el país con la finalidad de promover buenas prácticas para hacer frente a dicha problemática, se destaca problemas como Contaminación en las playas; Ausencia de rellenos sanitarios; Gestión de residuos sólidos; Caos ambiental urbano; Contaminación vehicular, esta problemática descrita, es consecuencia del comportamiento poco ecológico (Molina y De Durana., 2003; Fraj, 2002) de las personas, las cuales a pesar de conocer los problemas ambientales existentes en el mundo y en el país, asumen conductas que no contribuyen a mitigar el daño existente, y ello va desde las propias autoridades que no son rigurosos en implementar la normatividad ambiental existente, siendo uno de los principales factores, la poca concientización que se brinda a través de la educación, tanto en los colegios como en las universidades (Acebal y Brero, 2005; Martínez, 2008; Acebal, 2010; Gomera; Villamandos y Vaquero., 2012 ), puesto que es escasa la educación ambiental que se proporciona en dichas instituciones educativas. En los planes de estudio, de las escuelas de la Facultad de Ingeniería de la universidad privada de Tacna, se muestra que en la formación académica no se prioriza la concientización ambiental como un contenido transversal, lo cual podría influir en una actitud indiferente a priorizar el valorar y saber cuidar los recursos naturales regionales, generando que se formen profesionales sin un conocimiento claro de cómo hacer frente a los problemas ambientales.

Por tanto, la problemática planteada, tiene como una de sus principales causas, la poca formación educativa relacionada con el cuidado del nuestro medio ambiente y el bajo nivel de preocupación ambiental que debería caracterizar a todos los estudiantes en formación universitaria, de otra forma, de continuar dicha realidad, el problema ambiental se seguirá agudizando, a consecuencia del comportamiento de las personas.

\section{Objetivos}

Determinar la relación entre la preocupación ambiental y la conducta ecológica en estudiantes de la Facultad de Ingeniería de la Universidad Privada de Tacna. 


\section{Metodología}

El tipo de la investigación fue básico o puro, porque se busca aportar al conocimiento ya existente, de nivel correlacional, porque se analiza el comportamiento de las variables de estudio para luego relacionarlas, bajo un diseño descriptivo, donde las variables no son alteradas (Hernández, Fernández y Baptista, 2014). La investigación se efectuó con una muestra de 310 estudiantes de seis escuelas de la Facultad de Ingeniería de la Universidad Privada de Tacna (Civil 148; Sistemas 49; Electrónica 18; Ambiental 45; Industrial 38 y Agroindustrial 11), en el periodo de agosto a noviembre del año 2019. Las variables de investigación consistieron en medir la Preocupación ambiental como la predisposición para priorizar actividades en favor del cuidado del ambiente (Stern, 2000) con siete dimensiones (Alarma, confort, control doméstico, preocupación social, economía recibida, información y locus de control), además la variable Conducta ecológica, analizada como comportamientos sesgados a buscar proteger el medio ambiente (Corral, 2001) que constaba de seis dimensiones (Conservación de la energía, biodiversidad y recursos naturales, conservación del agua, consumo, movilidad y transporte y residuos). Se empleó un cuestionario para analizar el nivel de preocupación ambiental y otro para analizar la conducta ecológica, en ambos casos estructurados con una escala de Likert con cinco opciones de respuesta, donde: Completamente en desacuerdo o nunca (valor 1), en desacuerdo o casi nunca (valor 2), ni en desacuerdo ni de acuerdo o a veces (valor 3), de acuerdo o con frecuencia (valor 4), y completamente de acuerdo o siempre (valor 5); para ambos instrumentos.

Seguidamente fueron validados por expertos y para la confiabilidad, se aplicó una prueba piloto a 40 estudiantes, obteniéndose un valor del estadístico Alpha de Cronbach, para la variable "Preocupación ambiental" de 0,924 y "Conducta ecológica" de 0,880, indicadores que según George y Mallery (2003 son adecuados y pueden usarse para hacer el trabajo de campo.

Para el análisis de las dimensiones y las variables de estudio, las frecuencias de respuestas se agruparon en tres niveles: Bajo, regular y alto; para lo cual se generaron intervalos de igual amplitud, (Nivel bajo: 1,00 - 2,33; Nivel regular: 2,34 - 3,66 y Nivel alto: 3,67 - 5,00), posteriormente se aplicaron las siguientes técnicas estadísticas: Tablas de frecuencias, diagrama de barras, tablas cruzadas, estadística descriptiva (media aritmética y desviación estándar), prueba de Tukey y coeficiente de correlación Rho de Spearman.

\section{Resultados}

\section{La preocupación ambiental}

La variable preocupación ambiental es la disposición para priorizar actividades en favor del cuidado del ambiente (Stern, 2000), otros autores consideran como predictor del comportamiento ambiental y exploran otras dimensiones (Berenguer y Corraliza, 2000; berenger et al., (2001); Amerigo et al., 2012) pero convienen en general que está relacionado a las actitudes para el cuidado del medioambiente. En esa dirección los resultados encontrados que se categorizaron según niveles que se muestran en la tabla 1. 
Tabla 1

Categorización de la variable preocupación ambiental según sus dimensiones

\begin{tabular}{lcccccc}
\hline \multirow{2}{*}{ Dimensión } & \multicolumn{6}{c}{ Niveles } \\
\cline { 2 - 7 } & \multicolumn{3}{c}{ Bajo } & \multicolumn{2}{c}{ Regular } & \multicolumn{2}{c}{ Alto } \\
\cline { 2 - 7 } & Frecuencia & $\begin{array}{c}\text { Porcentaje } \\
(\%)\end{array}$ & Frecuencia & $\begin{array}{c}\text { Porcentaje } \\
(\%)\end{array}$ & Frecuencia & $\begin{array}{c}\text { Porcentaje } \\
(\%)\end{array}$ \\
\hline Alarma & 37 & 11,9 & 144 & 46,5 & 129 & 41,6 \\
Confort & 36 & 11,6 & 148 & 47,7 & 126 & 40,7 \\
Control doméstico & 35 & 11,3 & 137 & 44,2 & 138 & 44,5 \\
Preocupación social & 36 & 11,6 & 133 & 42,9 & 141 & 45,5 \\
Economía recibida & 34 & 11,0 & 127 & 41,0 & 149 & 48,0 \\
Información & 37 & 11,9 & 138 & 44,5 & 135 & 43,6 \\
Locus de control & 22 & 7,1 & 104 & 33,5 & 184 & 59,4 \\
Preocupación & 16 & 5,1 & 167 & 53,9 & 127 & 41,0 \\
ambiental & & & & & & \\
\hline
\end{tabular}

Nota. Adaptado del cuestionario "Preocupación ambiental” 2019.

Las dimensiones alarma que categoriza en tres niveles, resultó que el $46,5 \%$ de los estudiantes consideran que su nivel de preocupación por el impacto de la gravedad de la problemática ambiental es regular, el $41,6 \%$ considera que es de nivel elevado, y solamente el $11,9 \%$ que es de nivel bajo; al analizar por pregunta, se tiene que el aspecto más resaltado por los estudiantes es que consideran que las personas gastan más energía de la que necesitan tal como puede observase en la figura 1. Al se efectúa el análisis comparando las respuestas por escuelas profesionales, se encontró que los estudiantes de Ingeniería Ambiental $(82,2 \%)$ son los que se muestran más preocupados por los problemas ambientales, seguido por los de Ingeniería Industrial (51,3\%), siendo los menos alarmados los estudiantes de Ingeniería Electrónica (11,1 \%).

Respecto a la dimensión "Confort"; el 47,7 \% de los estudiantes consideran que su nivel de comodidad frente a la problemática ambiental es regular, el 40,7\% considera que es de nivel elevado, y solamente el $11,6 \%$ que es de nivel bajo; al analizar por pregunta, se tiene que el aspecto más resaltado por los estudiantes es que consideran que a las personas les preocupa más su comodidad que los problemas de calidad de vida en Tacna (figura 1). En el análisis comparativo por escuelas profesionales, se encontró que los estudiantes de Ingeniería Ambiental (84,4 \%) buscan ser más responsables con el aspecto ambiental a pesar que posiblemente se vea afectada su comodidad, seguido por los de Ingeniería Civil $(41,9 \%)$, siendo los estudiantes de Ingeniería Electrónica $(16,7 \%)$ los que menos destacan esta dimensión.

En la dimensión "Control doméstico"; el 44,5 \%, mostrado en la figura 1, consideran que su nivel de control doméstico sobre el uso responsable de la energía es elevada, el 44,2 \% considera que es de nivel regular, y solamente el $11,3 \%$ que es de nivel bajo; al analizar por pregunta, se tiene que el aspecto más resaltado por los estudiantes es que consideran que el consumo de energía en los hogares de cada familia es excesivo. De forma similar al efectuar el análisis comparado de las respuestas por escuelas, los estudiantes de Ingeniería Ambiental $(86,7 \%)$ resaltaron en la búsqueda de impulsar el control doméstico de los usos energéticos, seguidos por los de Ingeniería Civil $(48,6$ $\%)$, mientras que los estudiantes de Ingeniería Electrónica (33,3 \%) destacan menos esta dimensión. 
La dimensión "Preocupación social" representado en la figura 1; el 45,5\% de los estudiantes consideran que su nivel de preocupación social por los problemas ambientales es elevada, el 42,9\% considera que es de nivel regular, y solamente el $11,6 \%$ que es de nivel bajo; al analizar por pregunta, se tiene que el aspecto más resaltado por los estudiantes es que consideran que difunden noticias sobre los problemas ambientales para que su entorno familiar esté enterado. Al efectuar la comparación de las respuestas por escuelas, los estudiantes de Ingeniería Ambiental (88,9\%) resaltan su preocupación social, seguida por los de Ingeniería Civil $(45,3 \%)$, mientras que los estudiantes de Ingeniería Electrónica (11,1\%) destacan menos en esta dimensión.

Por otro lado, en la dimensión "Economía recibida" (figura 1); el 48,0 \% de los estudiantes consideran que su nivel de inversión económica que permita proteger el medio ambiente con su consumo diario es elevado, el $41,0 \%$ considera que es de nivel regular, y solamente el $11,0 \%$ que es de nivel bajo; al analizar por pregunta, se tiene que el aspecto más resaltado por los estudiantes es impulsar en su familia que se invierta más para cuidar el ambiente. Por otro lado comparando por escuelas, los estudiantes de Ingeniería Ambiental son quienes más invierten en adquirir cosas que no dañen el medio ambiente, seguido por los de Ingeniería Civil, siendo los estudiantes de Ingeniería Electrónica los que menos destacan esta dimensión. Los resultados se refieren a la dimensión "Información"; de donde el 48,0 \% de los estudiantes consideran que su nivel de información y estar al día sobre los problemas ambientales existentes es regular, el 43,6 \% considera que es de nivel elevado, y el $11,9 \%$ que es de nivel bajo; al analizar por pregunta, el aspecto más resaltado por los estudiantes es que buscan informarse sobre la problemática ambiental con relativa frecuencia, por otro lado el análisis comparando las respuestas por escuelas profesionales, los estudiantes de Ingeniería Ambiental son quienes más se muestran preocupación por informarse en temas ambientales, en contraposición a los estudiantes de Ingeniería Electrónica quienes menos destacan esta dimensión.

La dimensión "Locus de control" (figura 1); muestra que el 59,4 \% de los estudiantes consideran que su nivel de asumir el rol respecto a la crisis energética y ambiental es elevada, el 33,5\% considera que es de nivel regular, y solamente el 7,1\% que es de nivel bajo; al analizar por pregunta, se tiene que el aspecto más resaltado por los estudiantes es que consideran que se debería insertar en todas las carreras profesionales, temas o cursos afines para hacerle frente a los problemas ambientales y generar una mayor conciencia al respecto. En esta dimensión y de forma similar a las anteriores, los estudiantes de Ingeniería Ambiental asumen más el rol de preocupación frente a la problemática ambiental, en contraposición a los estudiantes de Ingeniería Electrónica que menos destacan.

La variable "Preocupación ambiental(figura 1)"; muestra que el 53,9\% de los estudiantes consideran que su nivel de preocupación por la problemática ambiental es regular, el 41,0\% considera que el nivel es elevada, y el 5,1 \% es bajo; al analizar por dimensión, resalta que el "Locus de control", seguido por la "Economía recibida", siendo la menos resaltada la "Alarma", mientras que Los estudiantes de Ingeniería Ambiental presentan mayores niveles de preocupación por la problemática ambiental, seguido por los de Ingeniería Industrial, siendo los estudiantes de Ingeniería Electrónica los que menos preocupados están. 


\section{Figura 1}

Representación de la dimensión preocupación ambiental: "Alarma", confort, control doméstico, preocupación social, economía percibida, información, locus de control y preocupación ambiental
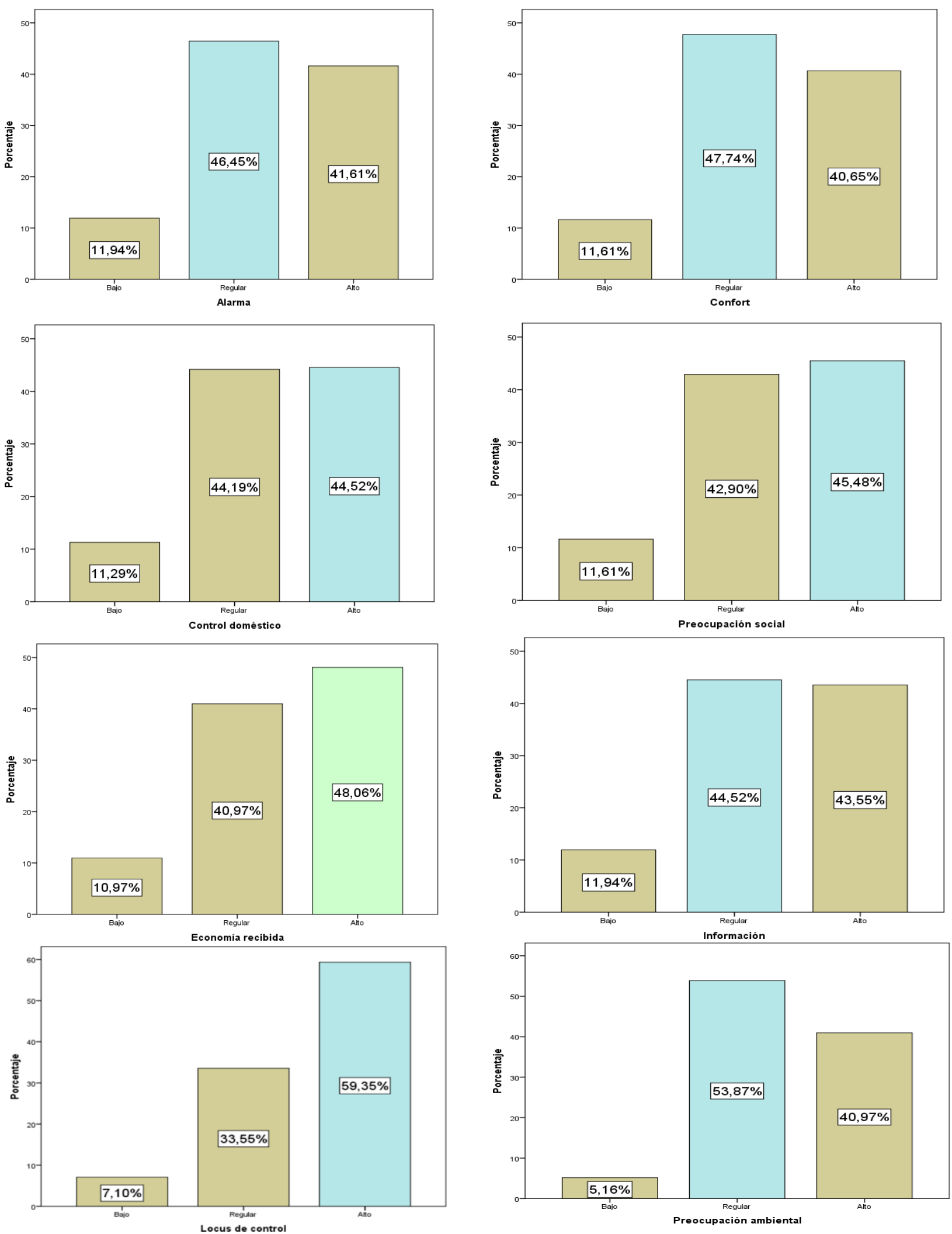

Nota. Adaptado del cuestionario "Preocupación ambiental” 2019. 


\section{La Conducta ecológica}

Varios autores (Hess et. Al., 1997; Corral, 2001; Berenguer, 2000), concuerdan que conducta ecológica son las acciones que buscan la protección ambiental, otros (stern, 2000; Bolzan, 2000) definen sus características como cambios en el consumo de recursos con la finalidad de conservar los recursos., por ello la investigación respecto a la conducta ecológica, desde la óptica de la conservación de energía, conservación del agua, residuos generados, biodiversidad y recursos naturales, entre otros, tal como se muestra en la tabla 2.

\section{Tabla 2}

Categorización de la variable Conducta ecológica según sus dimensiones

\begin{tabular}{|c|c|c|c|c|c|c|}
\hline \multirow{3}{*}{ Dimensión } & \multicolumn{6}{|c|}{ Niveles } \\
\hline & \multicolumn{2}{|c|}{ Bajo } & \multicolumn{2}{|c|}{ Regular } & \multicolumn{2}{|c|}{ Alto } \\
\hline & $\begin{array}{c}\text { Frecuen } \\
\text { cia }\end{array}$ & $\begin{array}{c}\text { Porcentaje } \\
\text { (\%) }\end{array}$ & Frecuencia & $\begin{array}{c}\text { Porcentaje } \\
\text { (\%) }\end{array}$ & Frecuencia & $\begin{array}{c}\text { Porcentaje } \\
\text { (\%) }\end{array}$ \\
\hline $\begin{array}{l}\text { Conservación de la } \\
\text { energía }\end{array}$ & 41 & 13,2 & 121 & 39,0 & 148 & 47,8 \\
\hline $\begin{array}{l}\text { Biodiversidad y } \\
\text { recursos naturales }\end{array}$ & 37 & 11,9 & 147 & 47,5 & 126 & 40,6 \\
\hline Conservación del agua & 43 & 13,9 & 179 & 57,7 & 88 & 28,4 \\
\hline Consumo & 31 & 10,0 & 139 & 44,8 & 140 & 45,2 \\
\hline Movilidad y transporte & 41 & 13,2 & 130 & 41,9 & 139 & 44,9 \\
\hline Residuos & 37 & 11,9 & 137 & 44,2 & 136 & 43,9 \\
\hline Conducta ecológica & 13 & 4,2 & 165 & 53,2 & 132 & 42,6 \\
\hline
\end{tabular}

Nota. Adaptado del cuestionario "Preocupación ambiental” 2019.

La dimensión "Conservación de la energía"; muestra que el 47,8\% de los estudiantes tienen un nivel alto de priorización por conservar la energía, el 39,0\% de nivel regular, el 13,2\% que es de nivel bajo; al comparar las respuestas por escuelas, estudiantes de Ingeniería Ambiental muestran un comportamiento más adecuado, seguido por los de Ingeniería Industrial, y los de Ingeniería Electrónica que menos destacan.

Respecto a la dimensión "Biodiversidad y recursos naturales"; de la variable Conducta Ecológica el $47,5 \%$ de los estudiantes muestran que su nivel de priorización por cuidar la biodiversidad y los recursos naturales es regular, el $40,6 \%$ considera que es de nivel alto, y el $11,9 \%$ es de nivel bajo; al analizar por pregunta, el aspecto más resaltado es que el Perú tenga parques nacionales y/o reservas naturales. Por otro lado el análisis comparado por respuestas, destaca de nuevo Ingeniería Ambiental, resaltando el cuidado de los recursos naturales, en comparación a las demás carreras.

En la "Conservación del agua"; el 57,7\% de estudiantes priorizan el uso racional del agua en un nivel regular, $28,4 \%$ considera como alto, y $13,9 \%$ de nivel bajo, mientras que al analizar comparativamente por escuelas; los estudiantes de Ingeniería Ambiental resaltan más la 
importancia de cuidar el agua, seguido por los de Ingeniería Civil, y los que menos destacan Ingeniería Industrial.

La dimensión "Consumo"; muestra en un nivel alto a 45,2 \% de estudiantes que indican que su consumo de productos no afectan al medio ambiente, mientras el $44,8 \%$ considera de nivel regular, y $10,0 \%$ de nivel bajo; al analizar por pregunta, el aspecto resaltado es el uso de focos ahorradores.

Los resultados de "Movilidad y transporte"; indican que el 44,9 \% de los estudiantes muestran un alto nivel de uso de formas de trasladarse que no afecten al medio ambiente, el 41,9\% considera que es regular, y el $13,2 \%$ es bajo; el aspecto más resaltado es la preferencia a utilizar el transporte público para movilizarse por la ciudad. En la comparación por escuelas, igual que en resultados anteriores, los estudiantes de Ingeniería Ambiental son quienes más resaltan en comparación a los de Ingeniería Electrónica.

En la dimensión "Residuos"; el 44,2\% aseguran que su nivel de frecuencia para implementar acciones de reciclaje de los residuos es regular, el $43,9 \%$ considera que es de nivel elevado, y el $11,9 \%$ que es bajo; al analizar por pregunta, se tiene que el aspecto más resaltado por los estudiantes es reúso de bolsas plásticas. También los que más destacan en esta dimensión son los estudiantes de Ingeniería Ambiental y en una posición opuesta se destaca a los estudiantes de Ingeniería Electrónica.

Los resultados se refieren a "Conducta ecológica"; el 53,2\% de los estudiantes muestran un nivel de conducta ecológica buscando priorizar el cuidado del medio ambiente de forma regular, el $42,6 \%$ considera como alto, y el 4,2\% de nivel bajo; el más resaltado fue la "Conservación de la energía", seguido de "Consumo", siendo el de menor valoración la "Conservación del agua". En el análisis comparando por escuelas profesionales, los estudiantes de Ingeniería Ambiental resaltan su conducta ecológica, seguido por los de Ingeniería Industrial, y los que menos destacan son estudiantes de Ingeniería Electrónica en conductas a favor del medio ambiente. 


\section{Figura 2}

Representación de la conducta ecológica y sus dimensiones: conservación de la energía, biodiversidad y recursos naturales, conservación del agua, consumo, movilidad y transporte, residuos y conducta ecológica.
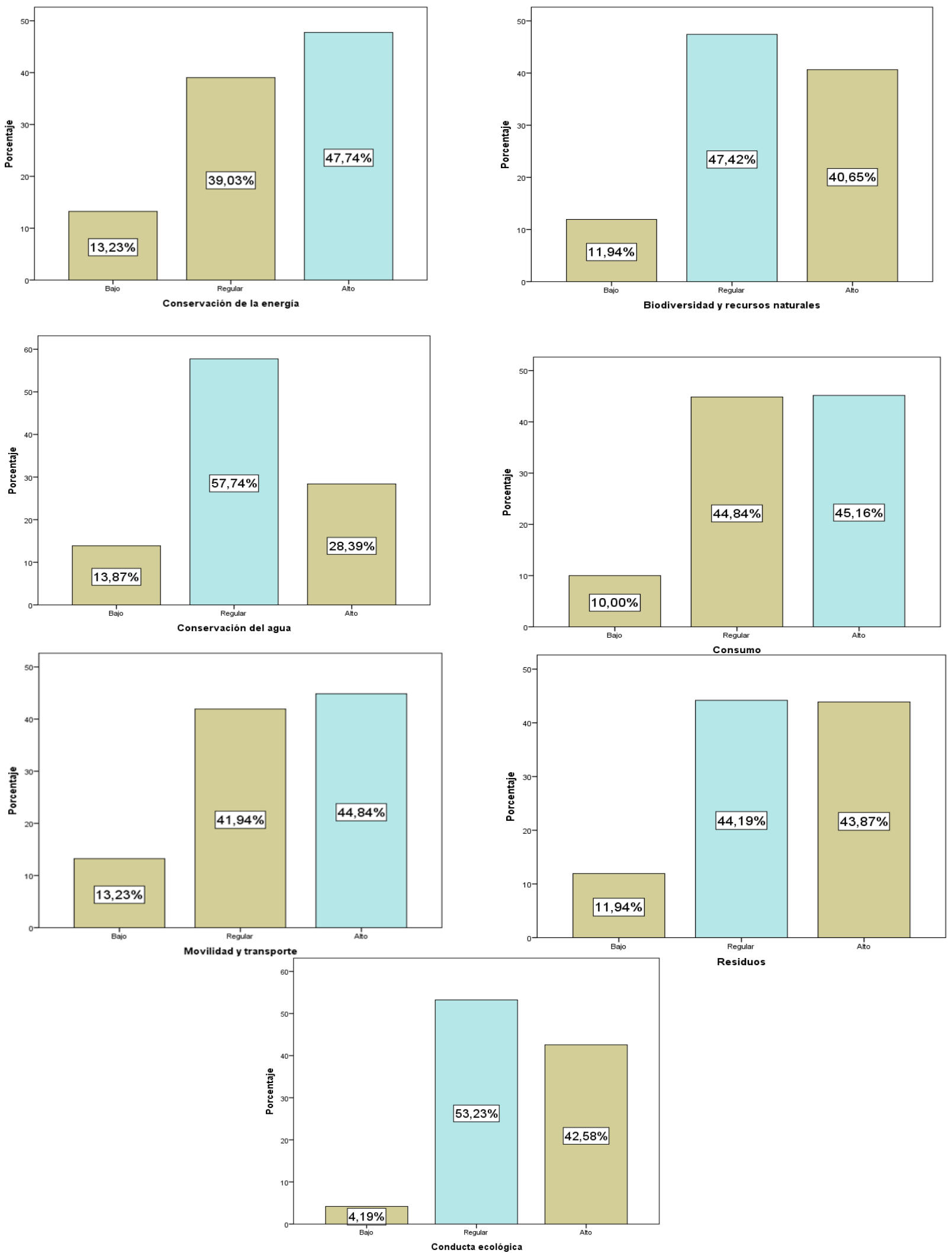

Nota. Adaptado del cuestionario "Preocupación ambiental” 2019. 


\section{Discusiones}

Los resultados obtenidos indican que existe relación directa y significativa entre la preocupación ambiental y la conducta ecológica en estudiantes de la Facultad de Ingeniería de la Universidad Privada de Tacna, puesto que se obtuvo un valor de Rho de Spearman de 0,614 y un valor de $p$ de 0,000; por tanto, una mejora en los niveles de preocupación ambiental en los estudiantes, donde la universidad priorice la inserción de cursos o unidades relacionadas con la problemática ambiental en los planes de estudio, generaría que el nivel de conducta ecológica mejoraría de forma significativa, con lo cual se estaría contribuyendo a que existan comportamientos en favor del cuidado de nuestros recursos naturales. Dichos resultados concuerdan con los encontrados por Montoya y Acevedo (2013) en su trabajo la "Preocupación ambiental entre población universitaria: Representaciones sociales e implicación personal en temas ambientales en la Universidad de Antioquia", quienes concluyen que existe un nivel elevado de preocupación ambiental, pero que se hace necesario fortalecer la formación universitaria en temas ambientales; también con los resultados encontrados por Gomera, Villamandos y Vaquero (2012) en su trabajo la "Medición y categorización de la conciencia ambiental del alumnado universitario: contribución de la universidad a su fortalecimiento", quienes concluyen que los estudiantes consideran que la universidad aún no ha logrado incorporar procesos efectivos que impulsen de forma concreta la ambientalización curricular, es decir que se consideren contenidos de cuidado del ambiente como conocimiento transversal a las carreras.

También existe concordancia con el trabajo de Carhuapoma y Juárez (2015) denominado los "Valores humanos, actitudes y comportamientos pro ambientales en estudiantes universitarios de Lima- Perú", quienes concluyen que existe relación directa y significativa entre los valores y las actitudes de los estudiantes; de forma similar con Chalco y Robles (2014) en su trabajo la "Educación ambiental y desarrollo de actitudes para la conservación del medio ambiente en los estudiantes de las instituciones educativas de la Red № 08, UGEL № 05 de SJL, Lima, 2014", quienes concluyen que existe relación directa y significativa entre la educación ambiental y las actitudes para conservar el ambiente.

Finalmente existe coincidencia con Almanza (2014) quien investigó la "Conciencia ambiental y el manejo de residuos pecuarios en el asentamiento humano Alto Tacna del Distrito Alto de la Alianza de la ciudad de Tacna", donde concluye que existe relación entre la conciencia ambiental y el mano de los residuos.

\section{Conclusiones}

Existe relación directa y significativa entre la preocupación ambiental y la conducta ecológica en estudiantes de la Facultad de Ingeniería de la Universidad Privada de Tacna, 2019; además la dimensión más destacada de la preocupación ambiental fue el "Locus de control”, y al comparar por escuelas profesionales, Ingeniería Ambiental presenta mayores valores medios; y la dimensión más destacada de la conducta ecológica fue la "Conservación de la energía".

El 53,9 \% de los estudiantes de la Facultad de Ingeniería de la Universidad Privada de Tacna consideran que su nivel de preocupación por la problemática ambiental es regular, el 41,0\% considera que es de nivel elevada, y solamente el 5,1 \% que es de nivel bajo. 
El 53,2 \% de los estudiantes de la Facultad de Ingeniería de la Universidad Privada de Tacna aseguran que su nivel de conducta ecológica buscando priorizar el cuidado del medio ambiente es regular, el $42,6 \%$ considera que es de nivel elevado, y solamente el $4,2 \%$ que es de nivel bajo.

\section{Referencias Bibliográficas}

Actualidad Ambiental. (2018, 6 febrero). Lanzan campaña de concientización: Juntos por el Medio Ambiente. https://www.actualidadambiental.pe/lanzan-campana-de-concientizacion-juntospor-el-medio-ambiente/.

Acebal Expósito, M. D. C. (2010). Conciencia ambiental y formación de maestras y maestros.

Acebal Expósito, M. D. C., \& Brero Peinado, V. (2005). Acerca de la conciencia ambiental de futuros formadores. Enseñanza de las Ciencias, (Extra), 1-5.

Martínez, A. G. (2008). La conciencia ambiental como herramienta para la educación ambiental: conclusiones y reflexiones de un estudio en el ámbito universitario. Universidad de Córdoba.

Hernández, R., Fernández, C., y Baptista, M. (2014). Metodología de la investigación. México: Editorial McGraw-Hill Educación. 5a Ed.

Amérigo, M., Aragonés, J. I., \& García, J. A. (2012). Explorando las dimensiones de la preocupación ambiental. Una propuesta integradora. Psyecology, 3(3), 299-311.

Berenguer, J., Corraliza, J. A., Martín, R., \& Oceja, L. V. (2001). Preocupación ecológica y acciones ambientales. Un proceso interactivo. Estudios de psicología, 22(1), 37-52.

Almanza, W. (2014). Conciencia ambiental y el manejo de residuos pecuarios en el asentamiento humano Alto Tacna del Distrito Alto de la Alianza de la ciudad de Tacna [tesis de maestro en gestión ambiental y desarrollo sostenible, Universidad Nacional Jorge Basadre Grohmann].

Repositorio institucional http://repositorio.unjbg.edu.pe/handle/UNJBG/992

Berenguer, J.; Corraliza, J.; Martín, R. y Oceja, L. (2000). Preocupación ecológica y acciones ambientales. Medio Ambiente y Comportamiento Humano, 22, 37-52.

Bolzan, C. (2008). Sistemas de gestión ambiental y comportamiento proambiental de trabajadores fuera de la empresa: aproximación de una muestra brasileña [Tesis de doctorado, Universidad de Barcelona].Repositorio institucional https://www.tdx.cat/handle/10803/2675

Carhuapoma, Y. y Juárez, P. (2015). Valores humanos, actitudes y comportamientos pro ambientales en estudiantes universitarios de Lima-Perú; artículo de la Universidad San Martín de Porres; revisado en http://www.aulavirtualusmp.pe/ojs/index.php/rpoe/article/view/737

Chalco, L. y Robles, L. (2014). Educación ambiental y desarrollo de actitudes para la conservación del medio ambiente en los estudiantes de las instituciones educativas de la Red № 08, UGEL № 05 de SJL, Lima, 2014 [tesis de doctor en administración de la educación, Universidad César Vallejo]. Repositorio institucional http://repositorio.ucv.edu.pe/bitstream/handle/UCV/9224/Chalco_RLNRobles_ALA.pdf?sequence $=1 \&$ isAllowed $=y$

Corral, V. (2001). Comportamiento proambiental. Una introducción al estudio de las conductas protectoras del medio ambiente. Santa Cruz de Tenerife, España: Resma. 
Corraliza, J. y Berenguer, J. (1998). Estructura de las actitudes ambientales: ¿Orientación general o especialización actitudinal? Revista de psicología social. 13 (3). pp. $399-406$.

Dietz, T., Stern, P. \& Guagnano, G. (1998). Social structural and social psychological bases of environmental concern. Environment and Behavior, 30, 450-471.

Fraj, E. (2002). Comportamiento del consumidor ecológico. Esic Editorial.

George, D. y Mallery, P. (2003). SPSS for Windows step by step: A Simple Guide and Reference. Boston: Allyn \& Bacon. 11.0, $4^{\circ}$ Edición.

Gomera, A.; Villamandos, F. y Vaquero, M. (2012). Medición y categorización de la conciencia ambiental del alumnado universitario: contribución de la universidad a su fortalecimiento; artículo de la revista de currículum y formación del profesorado (España); revisado en https://www.redalyc.org/pdf/567/56724395011.pdf

Hernández, R., Fernández, C., y Baptista, M. (2014). Metodología de la investigación. México: Editorial McGraw-Hill Educación. 5a Ed.

Hess, S.; Suárez, E. y Martínez, J. (1997). Estructura de la conducta ecológica responsable mediante el análisis de la Teoría de las Facetas. Revista de Psicología Social Aplicada, 7 (2/3).

Hines, J.; Hungerford, H. \& Tomera, A. (1986). Analysis and Synthesis of Research on Responsible Environmental Behavior: A Metaanalysis. Journal of Environmental Education, 2(18), 1-8.

Ministerio del Medio Ambiente - Chile. (2013). Comportamiento ambiental responsable de la ciudadanía; revisado en https://www.cepal.org/rio20/noticias/noticias/3/51573/03.Cristobal_d e_la_Maza.pdf

Molina, M. A. V., \& de Durana, C. A. E. G. (2003). Aproximación al perfil sociodemográfico del consumidor ecológico a través de la evidencia empírica: propuestas para el desarrollo del mix de marketing. Boletín económico de ICE, (2777).

Montoya, E. y Acevedo, E. (2013). Preocupación ambiental entre población universitaria: Representaciones sociales e implicación personal en temas ambientales en la Universidad de Antioquia; docentes de la Universidad de Antioquia; revisado en

file://C:/Users/Lenovo/Downloads/Dialnet- PreocupacionAmbientalEntrePoblacionUniversitaria5373055.pdf.Samdhal, D. \& Robertson, R. (1989). Social determinants of environmental concern. Specification and test of the model. Environment and Behaviour, 21. pp. 57-81.

Santana, H. (2017). Educación ambiental no formal y actitud hacia la conservación del medio ambiente de la población del C.P.M. Francisco Bolognesi de Tacna [tesis de maestro en gestión_ambiental y desarrollo sostenible, Universidad Nacional Jorge Basadre Grohmann].

Repositorio institucional http://repositorio.unjbg.edu.pe/handle/UNJBG/980

Scott, D y Willits, F. (1994). Environmental attitudes and behavior. A Pennsylvania survey. Environment and Behavior, 26, 239-260.

Stern, P. (2000). Toward a coherent theory of environmentally significant behavior. Journal of Social Issues, 56(3), 407-424. 